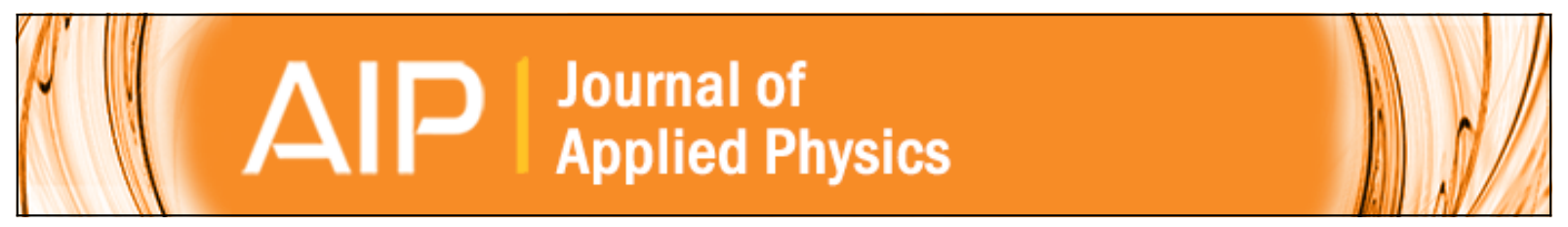

\title{
Enhanced domain contribution to ferroelectric properties in freestanding thick films
}

Jungho Ryu, Shashank Priya, Chee-Sung Park, Kun-Young Kim, Jong-Jin Choi, Byung-Dong Hahn, Woon-Ha

Yoon, Byoung-Kuk Lee, Dong-Soo Park, and Chan Park

Citation: Journal of Applied Physics 106, 024108 (2009); doi: 10.1063/1.3181058

View online: http://dx.doi.org/10.1063/1.3181058

View Table of Contents: http://scitation.aip.org/content/aip/journal/jap/106/2?ver=pdfcov

Published by the AIP Publishing

\section{Articles you may be interested in}

Structural changes and ferroelectric properties of BiFeO 3 - PbTiO 3 thin films grown via a chemical multilayer deposition method

J. Appl. Phys. 105, 014101 (2009); 10.1063/1.3053773

Scaling of structure and electrical properties in ultrathin epitaxial ferroelectric heterostructures

J. Appl. Phys. 100, 051609 (2006); 10.1063/1.2337363

Piezoelectric property of an epitaxial lead titanate thin film deposited by the hydrothermal method Appl. Phys. Lett. 88, 112908 (2006); 10.1063/1.2183364

Ferroelectric/ferroelastic behavior and piezoelectric response of lead zirconate titanate thin films under nanoindentation

J. Appl. Phys. 97, 074301 (2005); 10.1063/1.1870092

Piezoelectric properties of sputtered PbTiO 3 films: Growth temperature and poling treatment effects

J. Vac. Sci. Technol. A 16, 169 (1998); 10.1116/1.580954

\section{MIT LINCOLN} LABORATORY CAREERS

Discover the satisfaction of innovation and service to the nation
- Space Control

- Air \& Missile Defense

- Communications Systems \& Cyber Security

- Intelligence, Surveillance and

Reconnaissance Systems

\section{LINCOLN LABORATORY}

MASSACHUSETTS InSTITUTE OF TeChNOLOGY

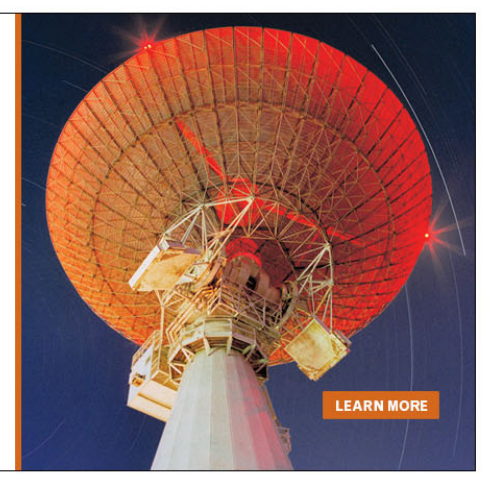




\title{
Enhanced domain contribution to ferroelectric properties in freestanding thick films
}

\author{
Jungho Ryu, ${ }^{1}$ Shashank Priya, ${ }^{2, a)}$ Chee-Sung Park, ${ }^{2}$ Kun-Young Kim, ${ }^{1,3}$ Jong-Jin Choi, ${ }^{1}$ \\ Byung-Dong Hahn, ${ }^{1}$ Woon-Ha Yoon, ${ }^{1}$ Byoung-Kuk Lee, ${ }^{1}$ Dong-Soo Park, ${ }^{1}$ and \\ Chan Park ${ }^{3}$ \\ ${ }^{1}$ Functional Ceramics Research Group, Korea Institute of Materials Science (KIMS), 66 Sangnam-Dong, \\ Changwon, Kyungnam 641-831, Republic of Korea \\ ${ }^{2}$ Department of Materials Science and Engineering, CEHMS, Virginia Polytechnic Institute and State \\ University, Blacksburg, Virginia 24061, USA \\ ${ }^{3}$ Division of Materials Science and Engineering, Pukyong National University, 100 Yong-Dang-Dong, \\ Nam-Gu, Busan 608-739, Republic of Korea
}

(Received 21 January 2009; accepted 20 June 2009; published online 28 July 2009)

\begin{abstract}
We report the success in fabricating clamped, "island," and freestanding $10 \mu \mathrm{m}$ thick piezoelectric films using aerosol deposition. The deposition was conducted at room temperature by impinging the piezoelectric particles flowing through the nozzle onto platinized silicon $\left(\mathrm{Pt} / \mathrm{Ti} / \mathrm{SiO}_{2} / \mathrm{Si}\right)$ substrate and crystallization was conducted by annealing at $700{ }^{\circ} \mathrm{C}$. Freestanding films were synthesized by increasing the cooling rate from annealing temperature to room temperature which resulted in large internal stress between the substrate and film interface. Dielectric and ferroelectric characterizations showed enhanced ferroelectric performance of freestanding films as compared to continuous or clamped film which was associated to increased domain contribution due to decrease in degree of clamping as further confirmed by piezoforce microscopy. (C) 2009 American Institute of Physics.

[DOI: $10.1063 / 1.3181058]$
\end{abstract}

\section{INTRODUCTION}

Piezoelectric composition given by the formulation $0.9 \mathrm{~Pb}\left(\mathrm{Zr}_{0.52} \mathrm{Ti}_{0.48}\right) \mathrm{O}_{3}-0.1 \mathrm{~Pb}\left(\mathrm{Zn}_{1 / 3} \mathrm{Nb}_{2 / 3}\right) \mathrm{O}_{3}$ (PZNT) exhibits interesting properties suitable for energy harvesting application. Recent investigations have shown that bulk polycrystalline ceramics of acceptor modified PZNT provides a giant magnitude of energy density characterized by the product of piezoelectric voltage constant and piezoelectric strain constant. ${ }^{1,2}$ With continuing emphasis on reduction in size of energy harvesting structures it is important to develop thick films with enhanced dielectric and piezoelectric properties. Thick films commonly synthesized by sol-gel techniques have been shown to be suitable for designing cost-effective transducer structures based upon cantilever mechanism. ${ }^{3}$ However, there are three fundamental limitations in further improvement of transducers characteristics, that is, (i) thickness of films using common deposition techniques is in the range of $2-5 \mu \mathrm{m}$, (ii) film is attached to substrate which requires lithography processes to pattern the transducer structure, and (iii) ferroelectric and piezoelectric properties of film are limited due to finite substrate clamping. These problems are the motivation behind our studies and here we report the success in synthesizing freestanding films with thickness of $10 \mu \mathrm{m}$. We further present the evidence illustrating the enhanced contribution from $180^{\circ}$ and non- $180^{\circ}$ domain wall displacements with reduced degree of clamping.

\footnotetext{
a) Author to whom correspondence should be addressed. Electronic mail: spriya@mse.vt.edu.
}

\section{EXPERIMENTAL}

High purity raw materials of $\mathrm{PbO}, \mathrm{TiO}_{2}, \mathrm{ZrO}_{2}, \mathrm{ZnO}$, and $\mathrm{Nb}_{2} \mathrm{O}_{5}$ (99.9\%, Sigma Aldrich Co., Milwaukee, WI) were used to prepare the starting powder of PZNT through conventional solid oxide route. The average particle size $\left(d_{50}\right)$ of the PZNT powder was $\sim 1.4 \mu \mathrm{m}$. Dried powders were used in aerosol deposition (AD) of thick films using the process described in detail elsewhere. ${ }^{4-6}$ The system consisted of a rectangular-shape nozzle with orifices of $5 \times 0.4 \mathrm{~mm}^{2}$ and dried air was used as carrier gas at a flow rate of $10 \mathrm{l} / \mathrm{min}$. The accelerated PZNT particles flowing through the nozzle collided with a platinized silicon $\left(\mathrm{Pt} / \mathrm{Ti} / \mathrm{SiO}_{2} / \mathrm{Si}\right)$ substrate and formed a dense ceramic film at RT which was crystallized by annealing at $700{ }^{\circ} \mathrm{C}$ for $1 \mathrm{~h}$ in air atmosphere. The deposited film area was of the dimension $\sim 5 \times 12 \mathrm{~mm}^{2}$ and the film thickness was controlled in the vicinity of $10 \mu \mathrm{m}$.

The AD mechanism is based upon the impact adhesion of fine particles accelerated with high kinetic energy as shown in Fig. 1(a), which results in high residual stresses in the synthesized films. ${ }^{4-8}$ The magnitude of internal stress is expected to increase with film thickness due to increased particle-particle interaction. Thus, a thicker film is more susceptible to detachment or cracking by external stresses, such as thermal stress induced by mismatch in thermal expansion coefficients between film and substrate materials. If the magnitude of interfacial stress is higher than the adhesion strength between film and substrate, the film will detach from substrate. We exploited this fact to synthesize freestanding PZNT films as shown in Fig. 1(a). The interfacial stress was controlled by changing the cooling rate during the annealing process. It was found that when the cooling rate was of the order of $-300{ }^{\circ} \mathrm{C} / \mathrm{h}$ than dense freestanding films 


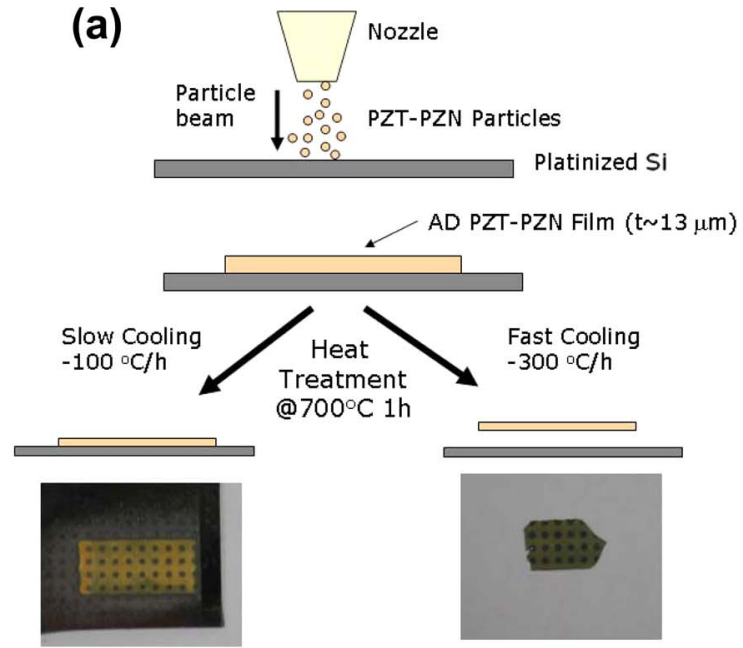

Clamped Thick Film

Freestanding Thick film

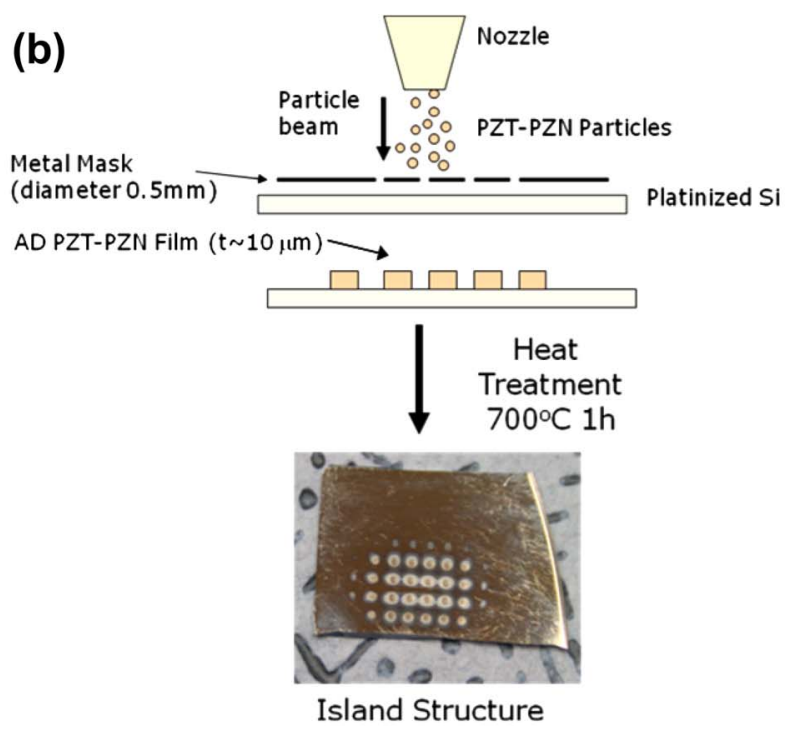

FIG. 1. (Color online) Schematics of the experimental process for synthesizing (a) clamped and freestanding thick films, and (b) island structure.

were obtained as confirmed by high magnification scanning electron microscopy (SEM) analysis conducted by scanning across the sample. This cooling rate was three times higher than that used for synthesizing the clamped (continuous film) AD film $\left(-100{ }^{\circ} \mathrm{C} / \mathrm{h}\right)$. In order to investigate the effect of degree of clamping on ferroelectric properties, we fabricated a partially clamped structure or "island" using the metal mask which had $0.5 \mathrm{~mm}$ diameter holes on top of substrate. Figure 1(b) schematically describes the modified AD process to deposit islands with a diameter of $500 \mu \mathrm{m}$. The thickness of islands was almost the same as that for fully clamped (continuous film) and freestanding films. The size of island was selected to be comparable with the clamped and freestanding film. After depositing top electrodes $(\mathrm{Pt})$ with similar diameter of $0.5 \mathrm{~mm}$ on the island by dc sputtering method, one can achieve various degrees of clamping as explained in Fig. 2. There were two reasons behind this selection: (i) to determine the degree of clamping, and (ii) to

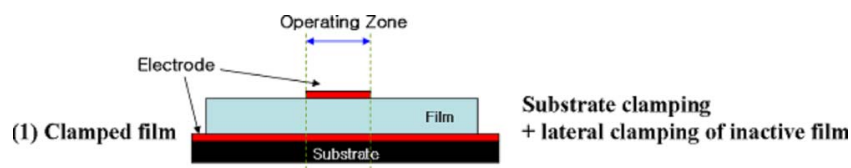

(2) island

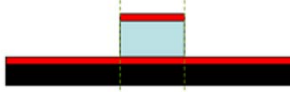

Substrate clamping

(3) Free standing

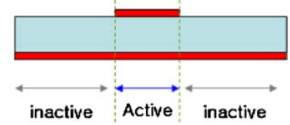

Lateral clamping of inactive film

FIG. 2. (Color online) Clamped, island, and freestanding films exhibiting two kinds of clamping, one arising from the substrate and other from the inactive area of film.

delineate the role of non- $180^{\circ}$ domain displacement. As illustrated in Fig. 2, when electrical properties of films are measured with locally deposited top electrodes than additional lateral clamping effect is present which is caused by inactive area of film. In other words, fully continuous film is clamped by the substrate and also from the inactive area of film. The island structure has only substrate clamping, while freestanding film has only lateral clamping from inactive area.

\section{RESULTS AND DISCUSSION}

Perovskite phase for starting powders and annealed films was confirmed by x-ray diffraction (XRD) (D-MAX 2200, Rigaku Co., Tokyo, Japan). Microstructure and thickness of films were monitored using field-emission scanning electron microscope (LEO Zeiss 1550). Figures 3(a) and 3(b) show the plane and cross-sectional views of clamped thick film. The microstructures of freestanding film are shown in Figs. 3(c) and 3(d). Both films were found to exhibit no trace of structural defects such as cracks, delaminations, and peelings. Figures 3(a) and 3(c) show the surface micrographs of films which reveals the dense microstructure with similar grain sizes. The thickness of films was measured to be $\sim 10 \mu \mathrm{m}$, as shown in Figs. 3(b) and 3(d). The clampedPZNT film was found to maintain good adhesion with the Pt-electroded Si substrate. From these figures it can be concluded that the film conditions were similar with only difference that one in attached to the substrate while the other is freestanding. Figure 3(e) shows the optical picture of the island structured film. Using high resolution XRD analysis, the residual stress in clamped films was estimated to be 114.0 $\pm 2.4 \mathrm{MPa}$ (tensile stress state), as shown in Fig. 2(f). It is evident from this data that the magnitude of internal stress is quite high, and this will have strong influence on the electrical properties.

The dielectric properties were measured as a function of frequency using Agilent 4294A impedance analyzer, and polarization hysteresis loops were obtained from a standardized P-LC100-K ferroelectric test system at RT. Figure 4 compares the dielectric and ferroelectric response of fully clamped, island, and freestanding thick films. Dielectric measurement as a function of frequency was conducted under the applied field of $E_{o}=0.5 \mathrm{kV}_{\mathrm{p}-\mathrm{p}} / \mathrm{cm}$. The low-field di- 

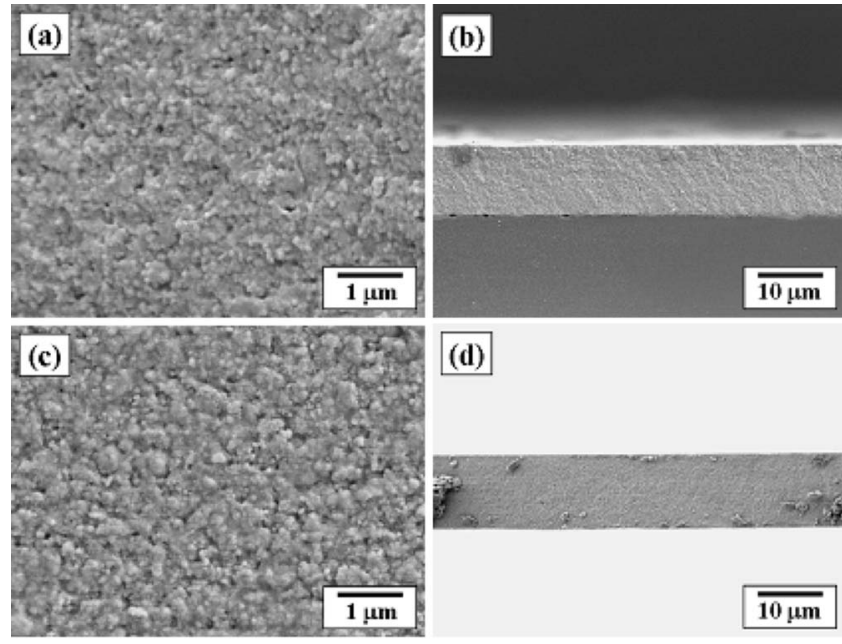

(d)
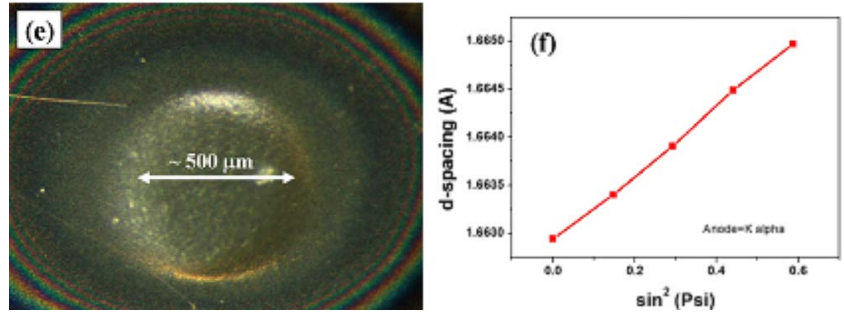

FIG. 3. (Color online) Plane and cross-sectional SEM images of [(a) and (b)] clamped and [(c) and (d)] freestanding thick films. (e) Optical microscopy picture of the island structure, and (f) Residual stress analysis of the clamped film shown in (a) and (b) using high resolution XRD. The stress was computed to be $114.0 \pm 2.4 \mathrm{MPa}$.

electric constant magnitude in Fig. 4(a) at $1 \mathrm{kHz}$ for fully clamped film was measured to be 1045 , which increased by $18.9 \%$ (1243) for the island structure, and $34.6 \%$ (1407) for the freestanding film. It can be noted that when lateral clamping from inactive area was removed the dielectric constant value increased by $18.9 \%$, while when the substrate clamping was removed the dielectric constant value increased by $34.6 \%$. We can summarize the results of Fig. 4 as (i) clamping from inactive area of film also influences the electrical properties, and (ii) effect of substrate clamping on electrical property is higher than other types of clamping. Based upon these observations, it is expected that when freestanding film has no lateral and substrate clamping then the dielectric constant value will be in the vicinity of 1605 (53\% increase).

Using the stress data from Fig. 3(f), one can approximate that rate of reduction in the magnitude of dielectric constant with increasing degree of clamping is -3.17 /MPa. Recently, dielectric measurements on the same composition have been conducted in bulk sintered ceramics by Islam and Priya, and the results showed dielectric constant in the range of 1700-2000. ${ }^{9}$ The increased magnitude of dielectric constant in bulk ceramics as compared to that of $\mathrm{AD}$ freestanding films can be explained on the basis of larger grain size in ceramics $(\sim 1 \mu \mathrm{m})$ as compared to $\sim 60-100 \mathrm{~nm}$ for the films, and more homogeneous microstructure. It is well known that both non- $180^{\circ}$ and $180^{\circ}$ domains walls contribute to the ferroelectricity. ${ }^{10-14}$ Large magnitude of dielectric constant in freestanding film clearly shows the increased domain contribution due to reduced clamping. The intermediate
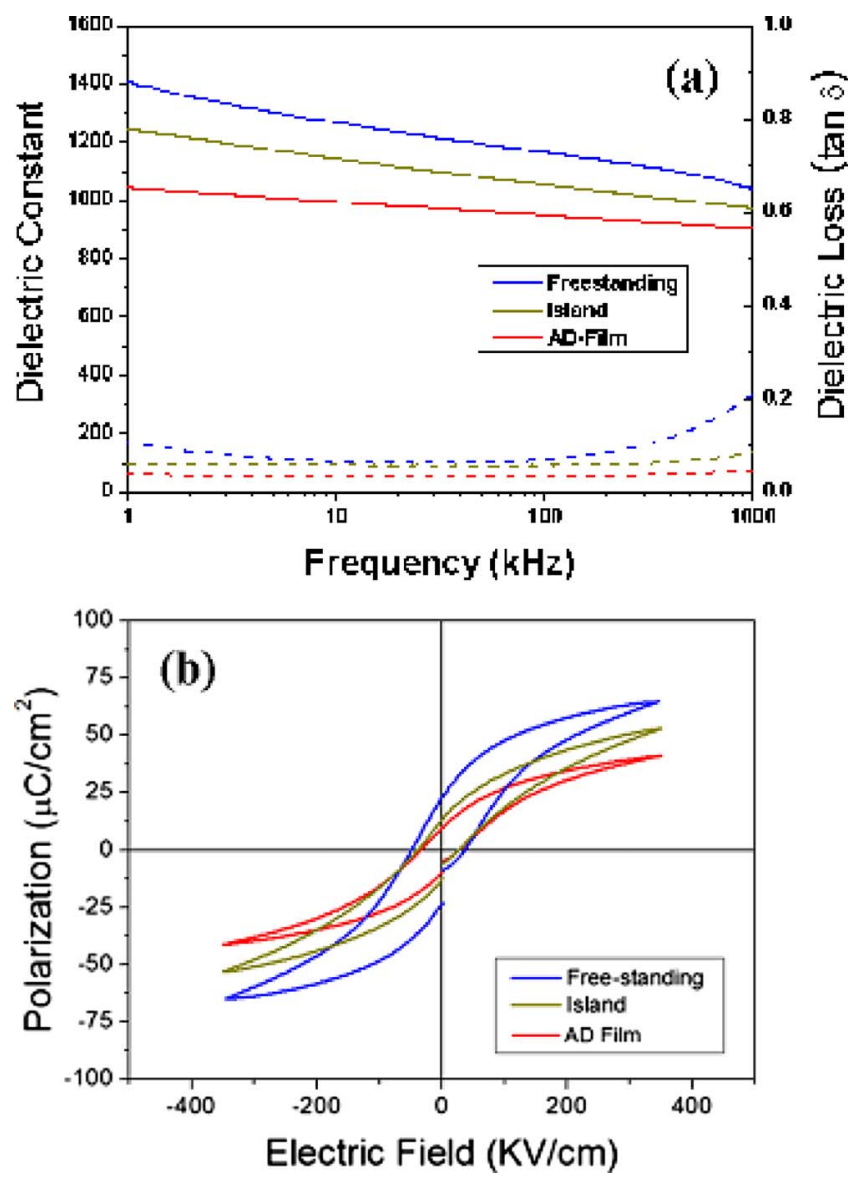

FIG. 4. (Color online) (a) Dielectric constant and loss of clamped, island, and freestanding thick films, (b) $P-E$ loop comparison between clamped, island, and freestanding thick films under the sweep of $360 \mathrm{kV} / \mathrm{cm}$ with frequency of $100 \mathrm{~Hz}$.

magnitude of dielectric constant in the island structure shows a systematic change in electrical property as the stress relaxation takes place. Using the dielectric data and boundary conditions, ${ }^{15}$ the degree of substrate clamping for the island structure was calculated to be in the vicinity of $55 \%$.

At low electric field drives one would only expect $180^{\circ}$ domain reversal as the switching of non- $180^{\circ}$ domains is accompanied by mechanical deformation and thus requires higher driving force. Recent results of Yimnirun et al. ${ }^{16}$ on bulk ceramics and thin films have shown that ferroelectric hysteresis at low fields follows the same scaling behavior $\langle A\rangle \propto f^{-1 / 3} E_{o}^{3}$, (where $\langle A\rangle$ is the hysteresis area, $\mathrm{f}$ is the frequency, and $E_{o}$ is the electric field amplitude) indicating that $180^{\circ}$ domain switching is prominent process under low drives.

Dielectric loss was found to follow the similar behavior, higher for freestanding film as compared to island and clamped. This further indicates the easiness in domain switching in freestanding films as compared to that of clamped ones. The origin of dielectric losses in ferroelectrics has been attributed to various factors such as vibrational and mechanical oscillations of $180^{\circ}$ and $90^{\circ}$ walls under electric field, dipolar reorientation leading to increment in the force constant of $90^{\circ}$ domain wall, interaction between $90^{\circ}$ domain walls and point defects as a possible energy-loss mechanism, and damping of the moving $90^{\circ}$ domain walls. ${ }^{10-14}$ Priya et 
$a l .{ }^{17,18}$ conducted extensive modeling of dielectric loss in relaxor-based solid solutions and shown that it is mainly composed of three components, $\chi_{\text {total }}^{\prime \prime}=\chi_{\text {domain }}^{\prime \prime}+\chi_{\text {inhomo }}^{\prime \prime}$ $+\chi_{\mathrm{PCE}}^{\prime \prime}$, where the three terms on right hand side are due to domain wall motion, excitation of structural irregularity and polarocaloric effect (PCE), respectively. Around room temperature the dielectric losses in poled relaxor-based materials under low-field external drives have significant contribution from excitation of structural irregularities. Thus, the results of Fig. 4(a) for dielectric loss can be explained based on the basis of increased domain contribution and from structural irregularities residing within the domain as the degree of clamping is reduced. It can also be seen in Fig. 4(a) that the dielectric loss for the clamped film are almost constant in the wide frequency range while that for freestanding and island structure show an increase at low frequency (below $10 \mathrm{kHz}$ ). This is an indication of finite non- $180^{\circ}$ domain contributions. The role of non- $180^{\circ}$ domains is mainly important at low frequencies as at higher frequencies there response will be clamped.

Further, evidence of the increased domain wall displacements can be seen in Fig. 4(b) which shows the P-E curve for fully clamped, island, and freestanding film. As expected from the dielectric response, the magnitude of remanant $\left(P_{r}\right)$ and saturation polarization was found to be highest for the freestanding film and then decreasing with increasing degree of clamping. Fitting of the remanant polarization data as a function of applied electric field in Fig. 4(b) resulted in following expressions:

$$
\begin{aligned}
P_{r}\left(\mu \mathrm{C} / \mathrm{cm}^{2}\right)= & 0.0867( \pm 0.0012) E_{o}(\mathrm{kV} / \mathrm{cm})+0.6 \\
& \times 10^{-4}\left( \pm 4.22 \times 10^{-6}\right) E_{o}^{2} \\
& (\text { freestanding }), \\
P_{r}\left(\mu \mathrm{C} / \mathrm{cm}^{2}\right)= & 0.0459( \pm 0.0028) E_{o}(\mathrm{kV} / \mathrm{cm})+0.5 \\
& \times 10^{-4}\left( \pm 9.91 \times 10^{-6}\right) E_{o}^{2} \quad \text { (clamped) }
\end{aligned}
$$

The lower magnitude of reversible permittivity and nonlinearity for the clamped films as compared to that of freestanding films further confirms the weaker domain wall contribution in the prior case. The enhancement of $2 \times$ in remanant polarization further indicates the contributions from $180^{\circ}$ and non- $180^{\circ}$ domains as piezoelectric constant is given by the expression: $d=2 \varepsilon Q P_{r}$, where $Q$ is the electrostriction constant. To further confirm this observation, we performed PFM measurement on the clamped and freestanding films as shown in Fig. 5. The evidence for increased domain wall displacements can be seen by comparing the Figs. 5(a) and 5(b) PFM images. The piezoelectric response of the freestanding film was significantly higher than that of the clamped film, as shown by the difference in contrast. This is an important result illustrating the enhanced piezoelectric activity in freestanding films. These freestanding films can be
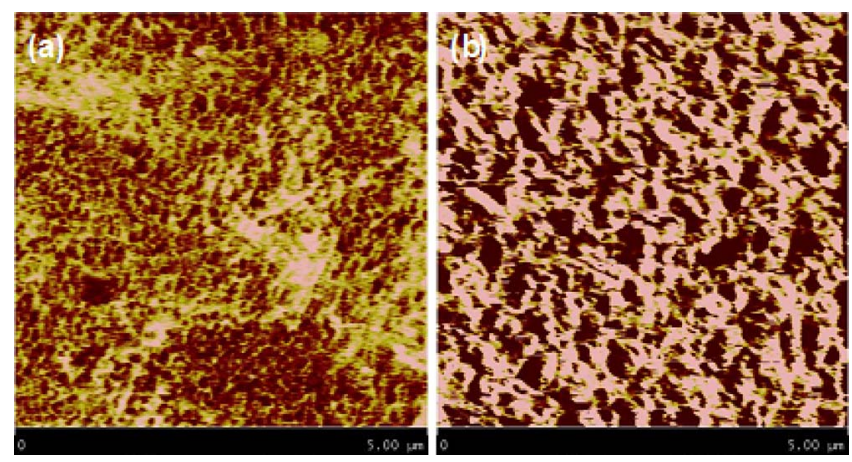

FIG. 5. (Color online) PFM images: (a) clamped thick films and (b) freestanding thick films.

utilized to fabricate transducer structures by using the regular microbonding and machining techniques.

\section{SUMMARY}

In summary, we have been successful in synthesizing the freestanding ferroelectric thick films using aerosol deposition. Using the same dimensions and electrode size for fully clamped, island structure, and freestanding films, we identified the effect of two different clamping conditions on electrical properties. Further, we have shown enhanced dielectric and ferroelectric performance in freestanding films which indicates the structural integrity of films and demonstrates the role of domain wall displacement.

\section{ACKNOWLEDGMENTS}

This work was financially supported by the Ministry of Knowledge Economy, Republic of Korea, through the Component-Material development program, and by the Office of Basic Energy Sciences, Department of Energy, USA.

${ }^{1}$ R. A. Islam and S. Priya, Appl. Phys. Lett. 88, 032903 (2006).

${ }^{2}$ R. Islam and S. Priya, J. Am. Ceram. Soc. 89, 3147 (2006).

${ }^{3}$ S. Priya, J. Electroceram. 19, 165 (2007).

${ }^{4}$ J.-J. Choi, J.-H. Jang, B.-D. Hahn, D.-S. Park, W.-H. Yoon, J. Ryu, and C. Park, J. Am. Ceram. Soc. 90, 3389 (2007).

${ }^{5}$ B.-D. Hahn, K.-H. Kim, D.-S. Park, J.-J. Choi, J. Ryu, W.-H. Yoon, C. Park, and D.-Y. Kim, Jpn. J. Appl. Phys., Part 1 47, 5545 (2008).

${ }^{6}$ J. Ryu, J.-J. Choi, B.-D. Hahn, D.-S. Park, W.-H. Yoon, and K.-H. Kim, Appl. Phys. Lett. 90, 152901 (2007).

${ }^{7}$ J. Akedo, J. Am. Ceram. Soc. 89, 1834 (2006).

${ }^{8}$ M. Lebedev and J. Akedo, Jpn. J. Appl. Phys., Part 1 41, 6669 (2002).

${ }^{9}$ R. Islam, S. Priya, and A. Amin, J. Mater. Sci. 42, 10052 (2007).

${ }^{10}$ K. W. Plessner, Proc. Phys. Soc. London 69, 1261 (1956).

${ }^{11}$ C. Kittel, Phys. Rev. 83, 458 (1951).

${ }^{12}$ G. Arlt and N. A. Pertsev, J. Appl. Phys. 70, 2283 (1991).

${ }^{13}$ V. S. Postnikov, V. S. Pavlov, and S. K. Turkov, J. Phys. Chem. Solids 31, 1785 (1970).

${ }^{14}$ J. O. Gentner, P. Gerthsen, N. A. Schmidt, and R. E. Send, J. Appl. Phys. 49, 4485 (1978).

${ }^{15}$ V. Nagarajan, A. Roytburd, A. Stanishevsky, S. Prasertchoung, T. Zhao, L. Chen, J. Melngailis, O. Auciello, and R. Ramesh, Nature Mater. 2, 43 (2003).

${ }^{16}$ R. Yimnirun, Y. Laosiritaworn, S. Wongsaenmai, and S. Ananta, Appl. Phys. Lett. 89, 162901 (2006).

${ }^{17}$ S. Priya and K. Uchino, Jpn. J. Appl. Phys., Part 1 42, 5158 (2003).

${ }^{18}$ S. Priya, K. Uchino, and D. Viehland, Appl. Phys. Lett. 80, 4217 (2002). 